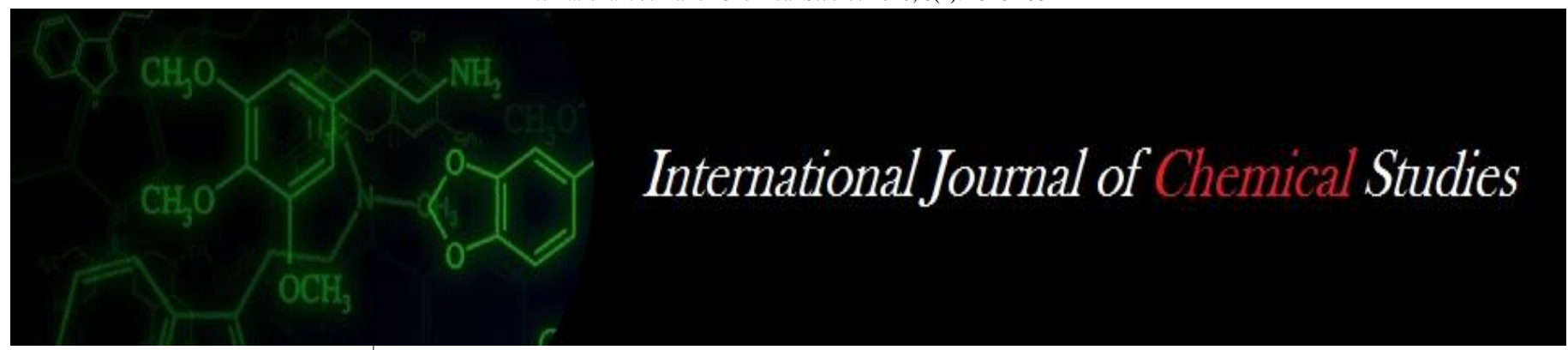

P-ISSN: 2349-8528

E-ISSN: 2321-4902

www.chemijournal.com

IJCS 2020; 8(2): 2348-2351

(C) 2020 IJCS

Received: 01-01-2020

Accepted: 03-02-2020

Raghubar Sahu

Krishi Vigyan Kendra, Banka,

Bihar, India

Dharmendra Kumar

Krishi Vigyan Kendra, Banka,

Bihar, India

Jubuli Sahu

Krishi Vigyan Kendra, Banka,

Bihar, India

Kumari Sharda

Krishi Vigyan Kendra, Barh,

Patna, Bihar, India

RK Sohane

Bihar Agricultural University, Sabour, Bhagalpur, Bihar, India

Corresponding Author: Jubuli Sahu

Krishi Vigyan Kendra, Banka,

Bihar, India

\section{Bio-efficacy of pre and post-emergence herbicides for control of complex weed flora in transplanted rice (Oryza sativa L.)}

\section{Raghubar Sahu, Dharmendra Kumar, Jubuli Sahu, Kumari Sharda and RK Sohane}

DOI: https://doi.org/10.22271/chemi.2020.v8.i2aj.9100

\begin{abstract}
A field experiment was conducted for two consecutive rainy seasons of 2017 and 2018 at Banka (Bihar) to evaluate the bio-efficacy of pre and post-emergence herbicides for control of complex weed flora in transplanted rice (Oryza sativa L.). The experimental field was infested with Echinochloa colona, Echinochloa crusgalli, Dactyloctenium aegyptium, Cynodon dactylon, Panicum repens, Ammania baccifera, Eclipta alba, Caesulia axillaris, Commelina benghalensis, Euphorbia hirta, Ludwigia parviflora, Cyperus iria, Cyperus difformis and Fimbristylis miliaceae. Density of grasses, broad-leaved weeds, sedges and total weeds at 60 DAT was recorded lower with pretilachlor $750 \mathrm{~g} / \mathrm{ha} f b 1$ hand weeding at 25 DAT and which was statistically comparable with butachlor $1500 \mathrm{~g} / \mathrm{ha} f b 1$ hand weeding at 25 DAT among weed management treatments excluding hands weeding twice at $20 \& 40$ DAT and weed free during both the years of experiment. Biomass of grasses, broad-leaved weeds, sedges and total weeds was registered less in pretilachlor $750 \mathrm{~g} / \mathrm{ha} f b 1$ hand weeding at 25 DAT and which was significantly superior to all weed management treatments excluding hands weeding twice at $20 \& 40$ DAT and weed free during both the years of experiment. More panicle weight, panicle length, grains/panicle, grain and straw yield was observed with application of pretilachlor $750 \mathrm{~g} / \mathrm{ha} f b 1$ hand weeding at 25 DAT.
\end{abstract}

Keywords: Bispyribac-Na, butachlor, pretilachlor, transplanted rice

\section{Introduction}

Rice is the world's most important food among all staple food crops and more than half of the world's population depends on rice for food, calories and protein, especially in developing countries. Rice suffers severely from weed competitions as compared to other cereal crops (Rao et al. 2007) ${ }^{[5]}$. Uncontrolled weeds compete with rice and cause yield losses to the tune of up to $76 \%$ in transplanted rice (Singh et al. 2004) ${ }^{[7]}$ and effective control of weeds had increased the grain yield by $85.5 \%$ (Mukherjee and Singh 2005) ${ }^{[2]}$. Transplanted rice faces diverse type of weed flora, consisting of grasses, broad-leaved weeds and sedges. They usually grow faster than rice and absorb available water, nutrient earlier than the rice and suppress rice growth. The farmers generally do 2-3 hands weeding in transplanted rice. For keeping weed population below threshold level, there is a need to evolve an effective integrated strategy of weed management involving chemical and mechanical methods. Herbicides appear to hold a great promise in dealing with effective, timely and economic weed suppression in transplanted rice. In recent past, several pre and post-emergence herbicides have been recommended for controlling weeds in transplanted rice. However, single application of one herbicide has seldom been found effective against complex weed flora throughout the critical period of competition. Pre-emergence herbicides such as pretilachlor, butachlor, oxadiargyl and anilofos are being frequently used for effective management of weeds in transplanted rice (Kumar et al. 2016) ${ }^{[9]}$. Use of herbicide along with the hand weeding is emerging out as very effective tool to tackle the problem of complex weed flora in transplanted rice. Integrated weed management systems have the potential to reduce herbicide use and to provide more robust weed management over the long term (Swanton and Weise, 1991) ${ }^{[10]}$. Keeping this in view, a field experiment was carried out to evaluate the bio-efficacy of pre and post-emergence herbicides for control of complex weed flora in transplanted rice. 


\section{Materials and Methods}

A field experiment was conducted during Kharif season of 2017 and 2018 at farmer's field of Banka District as an On Farm Trial to evaluate the bio-efficacy of pre and postemergence herbicides for control of complex weed flora in transplanted rice (Oryza sativa L.). The geographical location of the farm lies at $24^{\circ} 30^{\prime} \mathrm{N}$ latitude and $86^{\circ} 30^{\prime} \mathrm{E}$ latitude at an altitude of $79 \mathrm{~m}$ from the mean sea level. The soil of experimental site was sandy-clay-loam in texture with neutral $\mathrm{pH}$ value (7.37). It was low in organic $\mathrm{C}(0.45 \%)$ and available $\mathrm{N}(182.6 \mathrm{~kg} / \mathrm{ha})$, medium in available $\mathrm{P}(14.8 \mathrm{~kg} / \mathrm{ha})$ and $\mathrm{K}(210.8 \mathrm{~kg} / \mathrm{ha})$. The field experiment involving ten weed management treatments (weedy check, Hand weeding twice at 20 and 40 DAT, butachlor $1500 \mathrm{~g} / \mathrm{ha}$ pre-emergence, butachlor $1500 \mathrm{~g} / \mathrm{ha}$ pre-emergence $f b 1$ hand weeding at 25 DAT, butachlor $1500 \mathrm{~g} /$ ha pre-emergence $f b$ bispyribac-Na 25 $\mathrm{g} / \mathrm{ha}$ at $20 \mathrm{DAT}$, pretilachlor $750 \mathrm{~g} / \mathrm{ha}$ pre-emergence, pretilachlor $750 \mathrm{~g} /$ ha pre-emergence $f b 1$ hand weeding at 25 DAT, pretilachlor $750 \mathrm{~g} / \mathrm{ha}$ pre-emergence $f b$ bispyribac-Na $25 \mathrm{~g} / \mathrm{ha}$ at $20 \mathrm{DAT}$, bispyribac-Na $25 \mathrm{~g} / \mathrm{ha}$ at $20 \mathrm{DAT}$ and weed free) was conducted in a randomized block design replicated thrice with the rice cultivar 'Rajendra Sweta. Preemergence herbicides were sprayed in moist field within two days of transplanting and bispyribac-Na was sprayed at 20 DAT as follow-up. Treatment-wise pre and post-emergence herbicides were applied with knap-sack sprayer fitted with flat-fan nozzle using $375 \mathrm{~L} / \mathrm{ha}$ of water. Full dose of phosphorus (60 $\left.\mathrm{kg} \mathrm{P}_{2} \mathrm{O}_{5} / \mathrm{ha}\right)$, potassium $\left(60 \mathrm{~kg} \mathrm{~K} \mathrm{~K}_{2} \mathrm{O}\right)$ and half dose of nitrogen $(60 \mathrm{~kg} / \mathrm{ha})$ were applied at the time of sowing and remaining half dose of nitrogen $(60 \mathrm{~kg} / \mathrm{ha})$ was top dressed in two split doses after first and third irrigation. Rice was harvested at full physiological maturity, sun-dried for a week and threshed manually. Weed and crop samples were collected from each individual plot for studying various crop and weed characters. Weed samples were collected by placing a quadrat $(0.5 \mathrm{~m} \times 0.5 \mathrm{~m})$ randomly at two places in each plot. The data onweed density and biomass were subjected to square root transformation before statistical analysis to obtain homogeneity of variances. The data on density and biomass of weeds were taken at60 DAT and grain yield ( $\mathrm{t} / \mathrm{ha}$ ) was recorded at the time of harvesting. Analysis of two years done and comparisons were made at $5 \%$ level of significance.

\section{Results and Discussion \\ Weed flora}

The predominant weed flora of the experimental field was infested with grassy weeds, viz. Echinochloa colona, Echinochloa crusgalli, Dactyloctenium aegyptium, Commelina benghalensis, Cynodon dactylon and Panicum repens; broad-leaved weeds, viz. Ammania baccifera, Eclipta alba, Caesulia axillaris, Euphorbia hirta and Ludwigia parviflora; and sedges, viz. Cyperus iria,Cyperus difformis and Fimbristylis miliaceae during both the years of experimentation.

\section{Weed density and biomass}

All weed management treatments registered significantly superior to weedy check in the density and biomass of weeds during both the years of experimentation. Among the weed management treatments, pretilachlor $750 \mathrm{~g} / \mathrm{ha}$ pre-emergence (PRE) $f b 1$ hand weeding (HW) at 25 DAT effectively controlled grasses, broad-leaved weeds, sedges and total weed density at 60 DAT weed density (Table 1). This treatment was remained statistically at par with butachlor $1500 \mathrm{~g} / \mathrm{ha}$ (PRE) $f b 1 \mathrm{HW}$ at $25 \mathrm{DAT}$ excluding weed free and Hands weeding twice at 20 and 40 DAT during both the years of experimentation. This might be due to that pre-emergence herbicide application of pretilachlor suppress the early emerged weed and two hand weeding done at 25 DAT also reduced the population.

Application of pretilachlor $750 \mathrm{~g} / \mathrm{ha}$ (PRE) $\mathrm{fb} 1 \mathrm{HW}$ at $25 \mathrm{DAT}$ was registered reduced weed biomass of grasses, broad leaved weeds, sedges and it was found significantly superior as compared to all weed management treatments excluding weed free and hand weeding twice at 20 and 40 DAT during both the years of experiment. This might be due to suppression of initial emerging and emerged weeds on one hand and better crop growth on the other. The results are in agreement with the findings of (Singh et al. 2011 and Mohapatra et al. 2012) $[6,1]$. Among the herbicidal treatments, pretilachlor $750 \mathrm{~g} / \mathrm{ha}$ (PRE) $f b 1 \mathrm{HW}$ at $25 \mathrm{DAT}$ resulted in maximum weed control efficiency ( 86.24 and $86.20 \%$ ) followed by the application of butachlor $1500 \mathrm{~g} / \mathrm{ha}$ (PRE) $\mathrm{fb} 1 \mathrm{HW}$ at 25 DAT (84.17 and $84.12 \%)$ excluding weed free (100\% both the years) and two hands weeding at 20 and 40 DAT (95.13 and 95.10) (Table 2). This was due to integration of chemical and mechanical method of weed control resulting in broad-spectrum control of weeds.

\section{Yield attributes and yield}

Amongst weed control treatments, pretilachlor $750 \mathrm{~g} / \mathrm{ha}$ (PRE) $f b 1 \mathrm{HW}$ at 25 DAT resulted in more panicle weight, panicles length, grains/panicle, grain and straw yield and it was recorded significantly superior over all the weed management treatments excluding weed free and Hands weeding twice at 20 and 40 DAT during both the years of experiment. This might be due to effective control of weeds which in turn significantly increased the panicles/hill and grains/panicle consequently improving the grain yield. Control of weeds by herbicides during early stages of rice followed by hands weeding resulted in lowercompetition for growth resources, less weed competition for nutrients and better weed control that influenced the crop to grow better as evidenced in increased yield attributes. Similar findings were also observed by (Singh et al. 2005, Rajendran et al. 2003 and Ramachandra et al. 2012) ${ }^{[8,3,4]}$. The differences in 1000grain weight were non-significant due to weed management treatments. Weedy check recorded significantly lesser grain and straw yield amongst all the weed management treatments during both the years of experiment.

Table 1: Bio-efficacy of pre and post emergence herbicides on weed density for control of complex weed flora in transplanted rice (Oryza sativa L.)

\begin{tabular}{|c|c|c|c|c|c|c|c|c|}
\hline \multirow{3}{*}{ Treatment } & \multicolumn{8}{|c|}{ Weed density $\left(\right.$ no. $\left.^{-2}\right)$} \\
\hline & \multicolumn{2}{|c|}{ Grasses } & \multicolumn{2}{|c|}{ Broad leaved weeds } & \multicolumn{2}{|c|}{ Sedges } & \multicolumn{2}{|c|}{ Total weed density } \\
\hline & 2017 & 2018 & 2017 & 2018 & 2017 & 2018 & 2017 & 2018 \\
\hline Weedy check & $\begin{array}{c}7.76^{*} \\
(61.21)\end{array}$ & $\begin{array}{c}7.55 \\
(57.99)\end{array}$ & $\begin{array}{c}4.84 \\
(24.43)\end{array}$ & $\begin{array}{c}4.71 \\
(23.22)\end{array}$ & $\begin{array}{c}3.89 \\
(16.11)\end{array}$ & $\begin{array}{r}3.80 \\
(15.41\end{array}$ & $\begin{array}{c}10.02 \\
(101.35)\end{array}$ & $\begin{array}{c}9.89 \\
(98.81)\end{array}$ \\
\hline Hands weeding twice at 20 and 40 DAT & $\begin{array}{c}2.07 \\
(5.28) \\
\end{array}$ & $\begin{array}{c}1.97 \\
(4.87)\end{array}$ & $\begin{array}{c}1.43 \\
(3.04)\end{array}$ & $\begin{array}{c}1.39 \\
(2.93)\end{array}$ & $\begin{array}{c}0.89 \\
(1.80)\end{array}$ & $\begin{array}{c}0.87 \\
(1.76) \\
\end{array}$ & $\begin{array}{c}2.71 \\
(8.37)\end{array}$ & $\begin{array}{c}2.62 \\
(7.86)\end{array}$ \\
\hline
\end{tabular}




\begin{tabular}{|c|c|c|c|c|c|c|c|c|}
\hline Butachlor $1500 \mathrm{~g} / \mathrm{ha}(\mathrm{PE})$ & \begin{tabular}{|c|}
5.34 \\
$(29.58)$ \\
\end{tabular} & \begin{tabular}{|c|}
5.20 \\
$(28.05)$ \\
\end{tabular} & $\begin{array}{c}3.91 \\
(16.28) \\
\end{array}$ & $\begin{array}{c}3.81 \\
(15.48) \\
\end{array}$ & \begin{tabular}{|c|}
2.68 \\
$(8.16)$ \\
\end{tabular} & \begin{tabular}{|c|}
2.61 \\
$(7.82)$ \\
\end{tabular} & $\begin{array}{c}7.05 \\
(50.73) \\
\end{array}$ & $\begin{array}{c}6.92 \\
(48.88) \\
\end{array}$ \\
\hline Butachlor $1500 \mathrm{~g} / \mathrm{ha}(\mathrm{PE}) f b 1 \mathrm{HW}$ at $25 \mathrm{DAT}$ & $\begin{array}{c}3.77 \\
(15.18) \\
\end{array}$ & \begin{tabular}{|c|}
3.63 \\
$(14.17)$ \\
\end{tabular} & $\begin{array}{c}2.47 \\
(7.12) \\
\end{array}$ & $\begin{array}{c}2.41 \\
(6.80) \\
\end{array}$ & \begin{tabular}{|c|}
1.78 \\
$(4.18)$ \\
\end{tabular} & $\begin{array}{c}1.74 \\
(4.03) \\
\end{array}$ & $\begin{array}{c}4.70 \\
(23.10)\end{array}$ & $\begin{array}{c}4.57 \\
(21.88) \\
\end{array}$ \\
\hline Butachlor $1500 \mathrm{~g} / \mathrm{ha}(\mathrm{PE}) f b$ Bispyribac-Na $25 \mathrm{~g} / \mathrm{ha}$ & \begin{tabular}{|c|}
4.40 \\
$(20.41)$
\end{tabular} & \begin{tabular}{|c|}
4.28 \\
$(19.36)$ \\
\end{tabular} & $\begin{array}{c}3.35 \\
(12.21) \\
\end{array}$ & $\begin{array}{c}3.26 \\
(11.63) \\
\end{array}$ & \begin{tabular}{|c|}
2.18 \\
$(5.77)$ \\
\end{tabular} & \begin{tabular}{|c|}
2.13 \\
$(5.55)$ \\
\end{tabular} & $\begin{array}{c}5.76 \\
(34.15) \\
\end{array}$ & $\begin{array}{c}5.66 \\
(33.03) \\
\end{array}$ \\
\hline Pretilachlor $750 \mathrm{~g} / \mathrm{ha}(\mathrm{PE})$ & $\begin{array}{c}4.96 \\
(25.62)\end{array}$ & $\begin{array}{c}4.80 \\
(24.02)\end{array}$ & $\begin{array}{c}3.78 \\
(15.26)\end{array}$ & $\begin{array}{c}3.66 \\
(14.42)\end{array}$ & $\begin{array}{c}2.52 \\
(7.36)\end{array}$ & $\begin{array}{c}2.46 \\
(7.06)\end{array}$ & $\begin{array}{c}6.51 \\
(43.38)\end{array}$ & $\begin{array}{c}6.41 \\
(42.08)\end{array}$ \\
\hline Pretilachlor $750 \mathrm{~g} / \mathrm{ha}(\mathrm{PE}) f b 1 \mathrm{HW}$ at 2 & $\begin{array}{c}3.21 \\
(11.30) \\
\end{array}$ & $\begin{array}{c}3.10 \\
(10.61) \\
\end{array}$ & $\begin{array}{c}2.26 \\
(6.10) \\
\end{array}$ & $\begin{array}{c}2.19 \\
(5.80) \\
\end{array}$ & \begin{tabular}{|c|}
1.54 \\
$(3.39)$ \\
\end{tabular} & $\begin{array}{c}1.51 \\
(3.28) \\
\end{array}$ & $\begin{array}{c}4.29 \\
(19.43) \\
\end{array}$ & $\begin{array}{c}4.20 \\
(18.64) \\
\end{array}$ \\
\hline Pretilachlor $750 \mathrm{~g} / \mathrm{ha}(\mathrm{PE}) f b$ Bispyribac-Na $25 \mathrm{~g} / \mathrm{ha}$ at $20 \mathrm{DAT}$ & \begin{tabular}{|c|}
4.16 \\
$(18.36)$ \\
\end{tabular} & \begin{tabular}{|c|}
4.04 \\
$(17.31)$ \\
\end{tabular} & $\begin{array}{c}2.86 \\
(9.15) \\
\end{array}$ & $\begin{array}{c}2.77 \\
(8.67) \\
\end{array}$ & \begin{tabular}{|c|}
1.99 \\
$(4.98)$ \\
\end{tabular} & $\begin{array}{c}1.95 \\
(4.79) \\
\end{array}$ & $\begin{array}{c}5.26 \\
(28.66) \\
\end{array}$ & $\begin{array}{c}5.13 \\
(27.31) \\
\end{array}$ \\
\hline Bispyribac-Na $25 \mathrm{~g} / \mathrm{ha}$ at $20 \mathrm{DAT}$ & \begin{tabular}{|c|}
4.73 \\
$(22.37)$
\end{tabular} & $\begin{array}{c}4.59 \\
(22.08)\end{array}$ & $\begin{array}{c}3.64 \\
(14.24) \\
\end{array}$ & $\begin{array}{c}3.53 \\
(13.46) \\
\end{array}$ & \begin{tabular}{|c|}
2.36 \\
$(6.57)$ \\
\end{tabular} & $\begin{array}{c}2.30 \\
(6.30)\end{array}$ & $\begin{array}{c}6.22 \\
(39.71) \\
\end{array}$ & $\begin{array}{c}6.06 \\
(37.72) \\
\end{array}$ \\
\hline Weed free & $\begin{array}{c}1.00 \\
(0.00)\end{array}$ & $\begin{array}{c}1.00 \\
(0.00) \\
\end{array}$ & $\begin{array}{c}1.00 \\
(0.00)\end{array}$ & $\begin{array}{c}1.00 \\
(0.00)\end{array}$ & $\begin{array}{c}1.00 \\
(0.00)\end{array}$ & $\begin{array}{c}1.00 \\
(0.00)\end{array}$ & $\begin{array}{c}1.00 \\
(0.00)\end{array}$ & $\begin{array}{c}1.00 \\
(0.00)\end{array}$ \\
\hline $\operatorname{LSD}(\mathrm{p}=0.05)$ & 0.57 & 0.54 & 0.40 & 0.38 & 0.27 & 0.26 & 0.76 & 0.75 \\
\hline
\end{tabular}

*Data subjected to square root $(\sqrt{\mathrm{x}}+1)$ transformation and figures in parenthesis are original value, PE=Pre-emergence, DAT=Days after transplanting, $\mathrm{HW}=$ Hand weeding

Table 2: Bio-efficacy of pre and post emergence herbicides on weed biomass and weed control efficiency for control of complex weed flora in transplanted rice (Oryza sativa L.)

\begin{tabular}{|c|c|c|c|c|c|c|c|c|c|c|}
\hline \multirow{3}{*}{ Treatment } & \multicolumn{8}{|c|}{ Weed biomass $\left(\mathrm{g} \mathrm{m}^{-2}\right)$} & \multirow{2}{*}{\multicolumn{2}{|c|}{$\begin{array}{c}\text { WCE (\%) } \\
\begin{array}{c}\text { Total weed } \\
\text { density }\end{array}\end{array}$}} \\
\hline & \multicolumn{2}{|c|}{ Grasses } & \multicolumn{2}{|c|}{$\begin{array}{l}\text { Broad leaved } \\
\text { weeds }\end{array}$} & \multicolumn{2}{|c|}{ Sedges } & \multicolumn{2}{|c|}{ Total weed density } & & \\
\hline & 2017 & 2018 & 2017 & 2018 & 2017 & 2018 & 2017 & 2018 & 2017 & 2018 \\
\hline Weedy check & $\begin{array}{c}7.52 \\
(57.55)\end{array}$ & $\begin{array}{c}7.46 \\
(56.65)\end{array}$ & $\begin{array}{c}3.87 \\
(15.97)\end{array}$ & $\begin{array}{c}3.80 \\
(15.44)\end{array}$ & $\begin{array}{c}3.49 \\
(13.18)\end{array}$ & $\begin{array}{c}3.28 \\
(11.75)\end{array}$ & $\begin{array}{c}13.10 \\
(172.62)\end{array}$ & $\begin{array}{c}12.71 \\
(162.54)\end{array}$ & 0.00 & 0.00 \\
\hline Hands weeding twice at 20 and 40 DAT & $\begin{array}{c}0.54 \\
(1.29)\end{array}$ & $\begin{array}{c}0.53 \\
(1.28)\end{array}$ & $\begin{array}{c}0.22 \\
(1.04)\end{array}$ & $\begin{array}{c}0.20 \\
(1.04)\end{array}$ & $\begin{array}{c}0.18 \\
(1.03) \\
\end{array}$ & $\begin{array}{c}0.18 \\
(1.03)\end{array}$ & $\begin{array}{c}2.72 \\
(8.39)\end{array}$ & $\begin{array}{c}2.64 \\
(7.96)\end{array}$ & 95.13 & 95.10 \\
\hline Butachlor 1 & $\begin{array}{c}3.57 \\
(13.74)\end{array}$ & $\begin{array}{c}3.55 \\
(13.60)\end{array}$ & $\begin{array}{c}1.90 \\
(4.61)\end{array}$ & $\begin{array}{c}1.84 \\
(4.38)\end{array}$ & $\begin{array}{c}1.65 \\
(3.72) \\
\end{array}$ & $\begin{array}{c}1.53 \\
(3.34)\end{array}$ & $\begin{array}{c}9.09 \\
(83.62)\end{array}$ & $8.82(78.79)$ & 51.55 & 51.52 \\
\hline Butachlor $1500 \mathrm{~g} / \mathrm{ha}(\mathrm{PE}) f b 1 \mathrm{HW}$ at $25 \mathrm{DAT}$ & $\begin{array}{l}1.77 \\
(4.13)\end{array}$ & $\begin{array}{l}1.76 \\
(4.09)\end{array}$ & $\begin{array}{c}0.88 \\
(1.77)\end{array}$ & $\begin{array}{c}0.79 \\
(1.67)\end{array}$ & $\begin{array}{c}0.73 \\
(1.53) \\
\end{array}$ & $\begin{array}{c}0.71 \\
(1.50)\end{array}$ & $\begin{array}{c}5.13 \\
(27.31)\end{array}$ & $4.98(25.80)$ & 84.17 & 84.12 \\
\hline $\begin{array}{c}\text { Butachlor } 1500 \mathrm{~g} / \mathrm{ha} \text { (PE) } f b \text { Bispyribac-Na } 25 \\
\mathrm{~g} / \mathrm{ha} \text { at } 20 \mathrm{DAT}\end{array}$ & $\begin{array}{c}2.43 \\
(6.90)\end{array}$ & $\begin{array}{c}2.41 \\
(6.80)\end{array}$ & $\begin{array}{l}1.29 \\
(2.66)\end{array}$ & $\begin{array}{c}1.22 \\
(2.48)\end{array}$ & $\begin{array}{c}1.10 \\
(2.21) \\
\end{array}$ & $\begin{array}{c}1.01 \\
(2.02)\end{array}$ & $\begin{array}{c}6.78 \\
(46.96)\end{array}$ & $6.58(44.29)$ & 72.79 & 72.75 \\
\hline Pretilachlo & $\begin{array}{c}3.08 \\
(9.48)\end{array}$ & $\begin{array}{l}3.05 \\
(9.30)\end{array}$ & $\begin{array}{c}1.63 \\
(3.65)\end{array}$ & $\begin{array}{c}1.57 \\
(3.46)\end{array}$ & \begin{tabular}{|c|}
1.47 \\
$(3.16)$ \\
\end{tabular} & $\begin{array}{c}1.45 \\
(3.10)\end{array}$ & $\begin{array}{c}8.98 \\
(81.64)\end{array}$ & $8.71(76.86)$ & 52.70 & 52.71 \\
\hline Pretilachlor $750 \mathrm{~g} / \mathrm{ha}(\mathrm{PE}) f b 1 \mathrm{HW}$ at $25 \mathrm{DAT}$ & $\begin{array}{c}1.29 \\
(2.66)\end{array}$ & $\begin{array}{l}1.27 \\
(2.61)\end{array}$ & $\begin{array}{c}0.71 \\
(1.50)\end{array}$ & $\begin{array}{c}0.61 \\
(1.37)\end{array}$ & $\begin{array}{c}0.55 \\
(1.30) \\
\end{array}$ & $\begin{array}{c}0.53 \\
(1.28)\end{array}$ & $\begin{array}{c}4.77 \\
(23.75) \\
\end{array}$ & $4.63(22.43)$ & 86.24 & 86.20 \\
\hline $\begin{array}{c}\text { Pretilachlor } 750 \mathrm{~g} / \mathrm{ha}(\mathrm{PE}) f b \text { Bispyribac-Na } \\
25 \mathrm{~g} / \mathrm{ha} \text { at } 20 \text { DAT }\end{array}$ & $\begin{array}{c}2.17 \\
(5.70)\end{array}$ & $\begin{array}{c}2.15 \\
(5.62) \\
\end{array}$ & $\begin{array}{l}1.09 \\
(2.18)\end{array}$ & $\begin{array}{c}1.02 \\
(2.04)\end{array}$ & \begin{tabular}{|c|}
0.92 \\
$(1.84)$ \\
\end{tabular} & $\begin{array}{c}0.89 \\
(1.79) \\
\end{array}$ & \begin{tabular}{|c|}
6.44 \\
$(42.47)$ \\
\end{tabular} & $6.25(40.06)$ & 75.39 & 75.35 \\
\hline Bispyribac-Na $25 \mathrm{~g} / \mathrm{ha}$ at $20 \mathrm{DAT}$ & $\begin{array}{c}2.80 \\
(8.84)\end{array}$ & $\begin{array}{c}2.77 \\
(8.67)\end{array}$ & $\begin{array}{c}1.50 \\
(3.25)\end{array}$ & $\begin{array}{c}1.43 \\
(3.04)\end{array}$ & \begin{tabular}{|c|}
1.29 \\
$(2.66)$
\end{tabular} & $\begin{array}{c}1.18 \\
(2.39)\end{array}$ & $\begin{array}{c}8.60 \\
(74.96)\end{array}$ & $8.34(70.55)$ & 56.57 & 56.59 \\
\hline Weed free & $\begin{array}{c}1.00 \\
(0.00)\end{array}$ & $\begin{array}{c}1.00 \\
(0.00)\end{array}$ & $\begin{array}{c}1.00 \\
(0.00)\end{array}$ & $\begin{array}{c}0.00 \\
(1.00)\end{array}$ & $\begin{array}{c}0.00 \\
(1.00)\end{array}$ & $\begin{array}{c}0.00 \\
(1.00)\end{array}$ & $\begin{array}{c}0.00 \\
(1.00)\end{array}$ & $0.00(1.00)$ & 100 & 100 \\
\hline $\mathrm{LSD}(\mathrm{p}=0.05)$ & 0.38 & 0.35 & 0.22 & 0.20 & 0.16 & 0.15 & 0.79 & 0.77 & - & - \\
\hline
\end{tabular}

$\mathrm{PE}=$ Pre-emergence, DAT=Days after transplanting, $\mathrm{HW}=$ Hand weeding

Table 3: Bio-efficacy of pre and post emergence herbicides on yield attributes and yields for control of complex weed flora in transplanted rice (Oryza sativa L.)

\begin{tabular}{|c|c|c|c|c|c|c|c|c|c|c|c|c|}
\hline \multirow{3}{*}{ Treatment } & \multicolumn{8}{|c|}{ Yield attributes } & \multicolumn{4}{|c|}{ Yield $\left(\mathrm{t} \mathrm{ha}^{-1}\right)$} \\
\hline & \multicolumn{2}{|c|}{$\begin{array}{c}\text { Panicle weight } \\
\text { (g) }\end{array}$} & \multicolumn{2}{|c|}{$\begin{array}{c}\text { 1000-grain } \\
\text { weight }\end{array}$} & \multicolumn{2}{|c|}{$\begin{array}{c}\text { Panicle length } \\
\text { (cm) }\end{array}$} & \multicolumn{2}{|c|}{ Grains/panicle } & \multicolumn{2}{|c|}{ Grain } & \multicolumn{2}{|c|}{ Straw } \\
\hline & 2017 & 2018 & 2017 & 2018 & 2017 & 2018 & 2017 & 2018 & 2017 & 2018 & 2017 & 2018 \\
\hline Weedy check & 1.10 & 1.11 & 16.40 & 16.56 & 11.10 & 11.21 & 62.00 & 62.81 & 2.34 & 2.39 & 3.42 & 3.49 \\
\hline Hands weeding twi & 4.00 & 4.04 & 17.10 & 17.27 & 20.80 & 21.01 & 219.00 & 221.93 & 4.53 & 4.62 & 6.58 & 6.71 \\
\hline Butachlor $1500 \mathrm{~g} / \mathrm{ha}(\mathrm{PE})$ & 2.66 & 2.69 & 16.50 & 16.67 & 60 & 74 & 133.00 & 134.76 & 3.16 & 3.22 & 4.61 & 4.71 \\
\hline Butachlor $1500 \mathrm{~g} / \mathrm{ha}($ & 3.6 & & 16.90 & 97 & & 09 & & 203.70 & 4.03 & 4.11 & 5.88 & 6.00 \\
\hline $\begin{array}{c}\text { Butachlor } 1500 \mathrm{~g} / \mathrm{ha}(\mathrm{PE}) \mathrm{fb} \text { Bispyribac-Na } 25 \mathrm{~g} / \mathrm{ha} \\
\text { at } 20 \mathrm{DAT}\end{array}$ & 3.2 & 3. & 16.70 & 1 & 1 & 1 & 00 & 1 & 3.58 & 3.65 & 5.23 & 5.33 \\
\hline Pretilachlor $750 \mathrm{~g} / \mathrm{ha}(\mathrm{PE})$ & 2.87 & 2.90 & 16.50 & 16.67 & 14.90 & 15.05 & 145.00 & 146.95 & 3.31 & 3.38 & 4.85 & 4.95 \\
\hline Pretilachlor $750 \mathrm{~g} / \mathrm{ha}(\mathrm{PE}) \mathrm{fb} 1 \mathrm{HW}$ a & 3.80 & 3.84 & 17.00 & 17.17 & 19.50 & 19.70 & 211.00 & 213.84 & 4.27 & 4.36 & 6.23 & 6.36 \\
\hline $\begin{array}{c}\text { Pretilachlor } 750 \mathrm{~g} / \mathrm{ha}(\mathrm{PE}) f b \text { Bispyribac-Na } 25 \mathrm{~g} / \mathrm{ha} \\
\text { at } 20 \mathrm{DAT}\end{array}$ & 3.4 & 3.48 & 16.80 & 16.97 & 17.60 & 17.78 & 187.00 & 189.52 & 3.89 & 3.97 & 5.68 & 5.79 \\
\hline Bispyribac-Na $25 \mathrm{~g} / \mathrm{ha}$ at $20 \mathrm{DAT}$ & 3.00 & 3.03 & 16.60 & 16.77 & 15.40 & 15.55 & 160.00 & 162.14 & 3.44 & 3.51 & 5.02 & 5.12 \\
\hline Weed free & 4.30 & 4.34 & 17.30 & 17.47 & 21.20 & 21.41 & 227.00 & 229.99 & 4.84 & 4.94 & 7.07 & 7.21 \\
\hline $\operatorname{LSD}(\mathrm{p}=0.05)$ & 0.42 & 0.45 & NS & NS & 2.40 & 2.47 & 24.28 & 24.61 & 0.39 & 0.41 & 0.66 & 0.61 \\
\hline
\end{tabular}

$\mathrm{NS}=$ Non Significant, $\mathrm{PE}=$ Pre-emergence, DAT=Days after transplanting, HW=Hand weeding 


\section{Conclusion}

It may be concluded that, pretilachlor $750 \mathrm{~g} / \mathrm{ha}$ (PRE) $f b 1$ HW at 25 DAT was resulted in significantly higher grain yield and monetary return as well as found effective in managing weeds in transplanted rice.

\section{References}

1. Mohapatra PC, Din M, Parida BC, Patel SP, Mishra P. Effect of mechanical planting and weeding on yield, water-use efficiency and cost of production under modified system of rice intensification. Indian Journal of Agricultural Sciences. 2012; 82(3):280-283.

2. Mukherjee D, Singh RP. Effect of low doses of herbicides on weeds, nutrient uptake and yield of transplanted rice. Indian Journal of Agronomy. 2005; 50:194-196.

3. Rajendran R, Ravi V, Ramanathan S, Jayaraj T, Chandrasekaran B, Balasubramanian V. Evaluation of selected crop management technologies for enhancing rice productivity and profitability in Tamil Nadu, India. p. 25In: National Seminar on Advance in Agricultural Resource Management, 2003.

4. Ramachandra C, Shivakumar N, Rajanna MP, Kalyanamurthy KN. Effect of age of seedlings and weed management under SRI on yield of rice. Indian Journal of Weed Science. 2012; 44(1):50-52.

5. Rao AN, Mortimer AM, Johnson DE, Sivaprasad B, Ladha JK. Weed management in direct-seeded rice. Advances in Agronomy. 2007; 93:155-257.

6. Singh B, Malik RK, Yadav A, Nandal DP. Herbicide efficacy in direct-seeded rice with different methods under wet and dry conditions. Indian Journal of Weed Science of Agronomy. 2011; 57(1):38-42

7. Singh G, Singh VP, Singh M. Effect of almix and butachlor alone and in combination on transplanted rice and associated weeds. Indian Journal of Weed Science 2004; 36(1, 2):64-67.

8. Singh RP, Singh CM, Singh AK. Effect of crop establishment methods, weed management and split nitrogen application on weeds and yield of rice (Oryza sativa). Indian Journal of Agricultural Sciences. 2005; 75(5):285-287.

9. Suresh Kumar R, Ashoka Reddy Y, Ravichandran S. Effects of weeds and their management in transplanted rice- A review. International Journal of Research in Applied, Natural and Social Sciences. 2016; 4(11):165180.

10. Swanton CJ, Weise SF. Integrated weed management: The rationale and approach. Weed Technology 1991; 5:657-663. 